\title{
Simultaneous optimization of a heat-integrated coal-to-SNG/MeOH polygeneration process based on rigorous kinetic models
}

Yu Zhuang ${ }^{a, b}$, Mingxin Li ${ }^{a}$, Lei Zhang ${ }^{a}$, Linlin Liu ${ }^{a}$, Jian Du ${ }^{a,{ }^{*}}$, Shengqiang Shen ${ }^{b}$

a. Institute of Process Systems Engineering, School of Chemical Engineering, Dalian University of Technology, Dalian 116023, Liaoning, China

b. Key Laboratory of Liaoning Province for Desalination, School of Energy and Power Engineering, Dalian University of Technology, Dalian 116023, Liaoning, China 
To increase the readability of the manuscript, the longest and least essential tables are shown in this Supporting Information. Here, there are four tables, labeled as S1, S2, S3, S4. This information is available free of charge via the Internet at http://pubs.acs.org/.

Table S1. The derived optimal variables of WGS-MS-Methanation

\begin{tabular}{clll}
\hline Unit & Parameters & Base plant & Optimized plant \\
\hline \multirow{3}{*}{ WGS } & Tout $(\mathrm{Cl}) / \mathrm{K}$ & 473.15 & 463.49 \\
& $\operatorname{Tout}(\mathrm{H1}) / \mathrm{K}$ & 533.15 & 442.45 \\
& $F(M P) / \mathrm{kmol} / \mathrm{h}$ & 1095.11 & 613.68 \\
\hline \multirow{3}{*}{$\mathrm{MS}$} & $\operatorname{Tout}(\mathrm{C} 3) / \mathrm{K}$ & 493.15 & 493.44 \\
& $R R_{M S}$ & 0.78 & 0.89 \\
& $p / \mathrm{MPa}$ & 8.00 & 5.10 \\
\hline \multirow{5}{*}{ Methanation } & $S R_{S N G}$ & 0.70 & 0.75 \\
& $\operatorname{Tout}_{(\mathrm{C} 2) / \mathrm{K}}$ & 570.00 & 531.23 \\
& $R R_{S N G}$ & 0.80 & 0.76 \\
& $\operatorname{Tout}(\mathrm{H} 5) / \mathrm{K}$ & 570.00 & 560.37 \\
& $\operatorname{Tout}(\mathrm{H} 6) / \mathrm{K}$ & 550.00 & 587.22 \\
\hline
\end{tabular}

Table S2. The derived optimal variables of WHRSC

\begin{tabular}{clll}
\hline Unit & Parameters & Base plant & Optimized plant \\
\hline & $F(C 5) / \mathrm{kmol} / \mathrm{h}$ & 8299.33 & 10975.93 \\
& $F(C 8) / \mathrm{kmol} / \mathrm{h}$ & 1523.94 & 2054.19 \\
& $F(C 11) / \mathrm{kmol} / \mathrm{h}$ & 213.31 & 282.31 \\
& Tout $(C 7) / \mathrm{K}$ & 833.72 & 881.52 \\
WHRSC & Tout $(C 10) / \mathrm{K}$ & 573.52 & 637.30 \\
& Tout $(C 13) / \mathrm{K}$ & 474.98 & 535.18 \\
& $p(C 7) / \mathrm{MPa}$ & 12.50 & 13.99 \\
& $p(C 10) / \mathrm{MPa}$ & 4.50 & 6.40 \\
& $p(C 13) / \mathrm{MPa}$ & 0.50 & 0.72 \\
\hline
\end{tabular}

Table S3. Input and output exergy of the base plant and optimized plant

\begin{tabular}{llll}
\hline & Parameters & Base plant & Optimized plant \\
\hline \multirow{2}{*}{ Input $/ \mathrm{kW}$} & Gasify agent & $18,704.96$ & $18,704.96$ \\
& Coal & $1,350,110.33$ & $1,350,110.33$
\end{tabular}




\begin{tabular}{|c|c|c|c|}
\hline & Electricity in ASU & $36,546.29$ & $36,546.29$ \\
\hline & Electricity in methanation unit & 3393.98 & 2865.08 \\
\hline & Steam in Rectisol & $16,905.12$ & $16,845.51$ \\
\hline & Steam in WGS & 3354.99 & 1880.07 \\
\hline & Electricity in MS & 2581.73 & 861.49 \\
\hline & Electricity in Rectisol & $39,182.46$ & $39,197.16$ \\
\hline \multirow{6}{*}{ Output/kW } & Steam generated by GAS & $16,373.40$ & $16,373.40$ \\
\hline & Steam generated by MS & $24,978.43$ & $26,221.96$ \\
\hline & SNG & $548,272.28$ & $548,290.99$ \\
\hline & $\mathrm{MeOH}$ & $273,286.48$ & $276,598.50$ \\
\hline & Power generated by WHRSC & $46,307.45$ & $66,706.27$ \\
\hline & Power generated by boiler & 5658.30 & 4797.26 \\
\hline \multicolumn{2}{|c|}{ Overall exergy efficiency $/ \%$} & 61.26 & 63.48 \\
\hline
\end{tabular}

Table S4. The details of the obtained heat exchanger network configuration

\begin{tabular}{cll|cll}
\hline $\begin{array}{l}\text { No. of Heat } \\
\text { Exchangers }\end{array}$ & $\begin{array}{l}\text { Area } \\
/ \mathrm{m}^{2}\end{array}$ & \multicolumn{1}{l}{$\begin{array}{l}\text { Heat Load } \\
/ \mathrm{kJ} / \mathrm{h}\end{array}$} & $\begin{array}{l}\text { No. of Heat } \\
\text { Exchangers }\end{array}$ & $\begin{array}{l}\text { Area } \\
/ \mathrm{m} 2\end{array}$ & $\begin{array}{l}\text { Heat Load } \\
/ \mathrm{kJ} / \mathrm{h}\end{array}$ \\
\hline E1 & 6310 & $1.36 \mathrm{E}+08$ & $\mathrm{E} 33$ & 7647 & $3.49 \mathrm{E}+07$ \\
E2 & 3656 & $6.44 \mathrm{E}+07$ & $\mathrm{E} 34$ & 4123 & $2.22 \mathrm{E}+07$ \\
E3 & 2746 & $1.04 \mathrm{E}+07$ & $\mathrm{E} 35$ & 6543 & $3.18 \mathrm{E}+07$ \\
E4 & 771 & $1.09 \mathrm{E}+07$ & E36 & $3.94 \mathrm{E}-03$ & $1.59 \mathrm{E}+03$ \\
E5 & 1722 & $4.02 \mathrm{E}+07$ & E37 & 918 & $1.11 \mathrm{E}+07$ \\
E6 & 1122 & $2.27 \mathrm{E}+07$ & E38 & 657 & $4.18 \mathrm{E}+06$ \\
E7 & 398 & $6.73 \mathrm{E}+06$ & E39 & 1395 & $8.09 \mathrm{E}+06$ \\
E8 & 16 & $1.48 \mathrm{E}+06$ & E40 & 1044 & $5.65 \mathrm{E}+06$ \\
E9 & 623 & $4.86 \mathrm{E}+07$ & E41 & 444 & $4.20 \mathrm{E}+06$ \\
E10 & 17 & $1.36 \mathrm{E}+06$ & E42 & 552 & $7.19 \mathrm{E}+06$ \\
E11 & 1927 & $3.79 \mathrm{E}+07$ & E43 & 1613 & $2.34 \mathrm{E}+07$ \\
E12 & 557 & $8.40 \mathrm{E}+06$ & E44 & 340 & $9.32 \mathrm{E}+06$ \\
E13 & 773 & $6.40 \mathrm{E}+06$ & E45 & 3243 & $1.31 \mathrm{E}+07$ \\
E14 & 17 & $1.36 \mathrm{E}+06$ & E46 & 88 & $1.31 \mathrm{E}+06$ \\
E15 & 841 & $3.10 \mathrm{E}+07$ & E47 & 4880 & $1.89 \mathrm{E}+07$ \\
E16 & 6 & $3.82 \mathrm{E}+05$ & E48 & 629 & $4.24 \mathrm{E}+06$ \\
E17 & 272 & $5.58 \mathrm{E}+06$ & E49 & 918 & $9.12 \mathrm{E}+06$ \\
E18 & 2305 & $1.31 \mathrm{E}+07$ & E50 & 391 & $4.81 \mathrm{E}+06$ \\
E19 & 342 & $4.86 \mathrm{E}+06$ & E51 & 995 & $4.55 \mathrm{E}+06$ \\
E20 & 207 & $6.74 \mathrm{E}+06$ & E52 & 72 & $1.72 \mathrm{E}+07$ \\
E21 & 3011 & $1.65 \mathrm{E}+07$ & E53 & 1223 & $6.82 \mathrm{E}+06$ \\
E22 & 282 & $2.05 \mathrm{E}+06$ & E54 & 421 & $1.68 \mathrm{E}+06$ \\
E23 & 167 & $1.38 \mathrm{E}+06$ & E55 & 232 & $3.28 \mathrm{E}+06$ \\
E24 & 34 & $1.96 \mathrm{E}+06$ & E56 & 7755 & $4.14 \mathrm{E}+07$ \\
E25 & 1142 & $4.63 \mathrm{E}+06$ & E57 & 7680 & $2.41 \mathrm{E}+07$ \\
& & & & &
\end{tabular}




\begin{tabular}{lll|lll} 
E26 & 19 & $6.55 \mathrm{E}+05$ & $\mathrm{E} 58$ & 1817 & $1.22 \mathrm{E}+07$ \\
E27 & 1397 & $9.94 \mathrm{E}+06$ & E59 & 122 & $2.60 \mathrm{E}+07$ \\
E28 & 2862 & $1.71 \mathrm{E}+07$ & E60 & 27 & $5.84 \mathrm{E}+06$ \\
E29 & 39 & $6.66 \mathrm{E}+05$ & E61 & 188 & $4.10 \mathrm{E}+07$ \\
E30 & 72 & $3.36 \mathrm{E}+06$ & E62 & 178 & $4.04 \mathrm{E}+07$ \\
E31 & 3051 & $5.80 \mathrm{E}+07$ & E63 & 101 & $2.44 \mathrm{E}+07$ \\
E32 & 823 & $1.78 \mathrm{E}+07$ & & & \\
\hline
\end{tabular}

